\title{
The tests of CP and CPT symmetry using the J-PET detector
}

\author{
Muhsin Mohammed ${ }^{1,2, *}$ and Aleksander Gajos ${ }^{1}$ \\ for the J-PET Collaboration \\ ${ }^{1}$ Institute of Physics, Jagiellonian University, ul. Łojasiewicza 11, 30-348 Cracow, Poland \\ ${ }^{2}$ Department of Physics, College of Education for Pure Sciences, University of Mosul, Mosul, Iraq
}

\begin{abstract}
Symmetries under the parity transformation $(\mathrm{P})$, charge-conjugation (C) and time reversal (T) are of fundamental importance in nuclear and elementary particle physics. Studies of the observables violating the combined CP symmetry constitute precise tests of the Standard Model. However, CP violation was observed to date only for systems involving quarks, raising the importance of searches its manifestations e.g. in purely leptonic systems. The $3 \gamma$ decay of spin-aligned ortho-positronium atoms (o-Ps) can be used to test $\mathrm{CP}$ invariance in such a purely leptonic system. The Jagiellonian Positron Emission Tomograph (J-PET) detection system enables experimental tests of CP and CPT through measurement of the expectation values of angular correlation operators odd under these transformations and constructed from (i) spin vector of the ortho-positronium atom, (ii) co-planar momentum vectors of photons originating from the decay of the positronium atom, and (iii) linear polarization direction of annihilation photons. Precise experimental symmetry tests with J-PET are possible thanks to a dedicated reconstruction technique of $3 \gamma$ ortho-positronium decays and a positronium production chamber including a highly porous aerogel target, whose setup allows for determining the orthopositronium spin polarization without the use of an external magnetic field.
\end{abstract}

\section{Introduction}

A novel detector based on strips of polymer scintillators is being developed by the J-PET Collaboration $[1,2]$. The J-PET detector has high timing properties which allow us to investigate the fundamental physics phenomena like discrete symmetries in the purely leptonic system [3]. One of the unique features of the J-PET detector is its ability to determine the spin of the positron and the formed positronium on an event by event basis.

The J-PET detector consists of 192 (EJ-230) plastic scintillator strips of dimensions $500 \times 19 \times 7 \mathrm{~mm}^{3}$, which are forming three concentric layers (48 modules of $425 \mathrm{~mm}$ radius, 48 modules of $467.5 \mathrm{~mm}$ radius and 96 modules of $575 \mathrm{~mm}$ radius [4]). Besides medical imaging applications [5-7], J-PET enables measurements of the momenta and the polarization vectors of the annihilation photons [3].

J-PET allows for registration of the decays of o-Ps into three gamma photons (o-Ps $\rightarrow$ $3 \gamma$ ) providing a high angular and energy resolution of $\sigma(\theta) \approx 0.4^{\circ}$ and $\sigma(E) \approx 4.1 \mathrm{keV}$,

\footnotetext{
*e-mail: muhsin.m@doctoral.uj.edu.pl
} 
respectively [8]. The preliminary reconstruction results indicate that the current version of J-PET detector gives a realistic chance to improve the best present limits established for the both $\mathrm{CP}$ and $\mathrm{CPT}$ symmetry violation tests in the decays of positronium atom by more than one order of magnitude.

\section{The study of $2 \gamma$ and $3 \gamma$ annihilations with J-PET}

A test measurement with the J-PET detector was carried out with an aluminum annihilation chamber implementing the scheme described in Ref. [9]. The chamber was shaped as a cylinder with a diameter of about $140 \mathrm{~mm}$. In the center of the chamber, a positron source was located in the form of ${ }^{22} \mathrm{Na}$ point-like radioactive source enclosed by two layers of Kapton foil glued together by an aluminum holder. A vacuum system connected with one of the cylindrical chamber bases ensured pressure at a level of about $8 \mathrm{~Pa}$. The walls of the chamber used in this test measurement did not contain any porous material for positronium production. Although the probability of o-Ps formation in aluminum is negligible, the direct electronpositron annihilation into three photons is possible and may be used to test the procedures for identification and reconstruction of such events regardless of the origin of the $3 \gamma$ state.
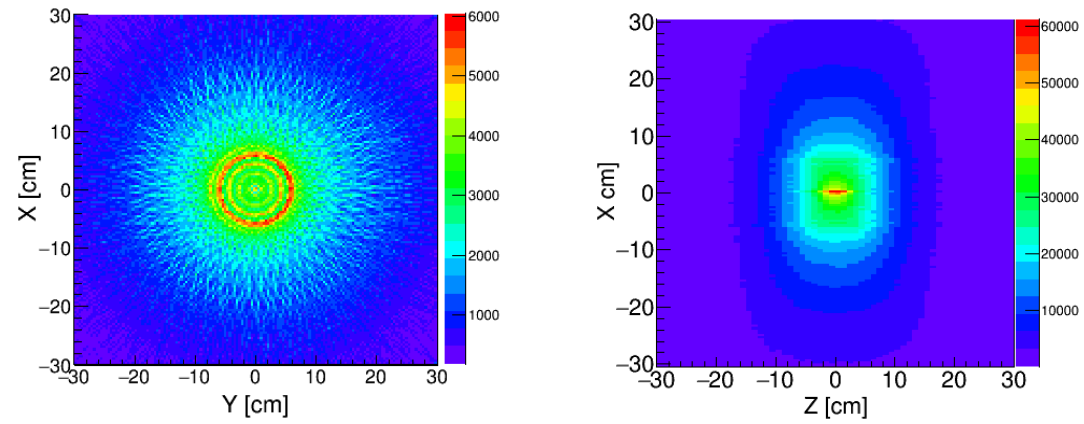

Figure 1. Tomographic image of the annihilation target cylinder obtained using Ps $\rightarrow 2 \gamma$ annihilations used as a benchmark for the 3 annihilation reconstruction studies. In the transverse view (left) the $|z|>3 \mathrm{~cm}$ region was excluded to avoid high intensity of annihilations occurring directly in the $\beta^{+}$source, visible in the side view (right).

To benchmark the reconstruction capabilities for $3 \gamma$ annihilations, the abundant $2 \gamma$ events were used. The selection criteria have been set as that only event candidates with two back to back $\gamma$ interactions were chosen with the azimuthal angle between the two hit detector modules in the range of $180 \pm 20^{\circ}$ and having the time over threshold of the recorded PMT signals (which is used to measure deposited energy in J-PET [10]) between $10 \mathrm{~ns}$ and $30 \mathrm{~ns}$, corresponding to energies deposited by $511 \mathrm{keV}$ photons in Compton scattering. Such selection allowed for the reduction of the raw data by about $99 \%$.

Figure 1 presents the image of the annihilation chamber obtained using the reconstructed $2 \gamma$ annihilation points. It is visible that the cylindrical chamber is well within the detector sensitive area in the transverse plane whereas the sensitive region along the $z$-axis spans about $8 \mathrm{~cm}$ from the detector center. As expected from the $2 \gamma$ benchmark results, reconstruction of direct annihilations into 3 photons was also demonstrated to be feasible using a trilaterationbased method [11] and its results from the test run are contained in Ref. [12].

Background events coming from accidental coincidences of a back-to-back annihilation with a third photon as well as secondary scatterings in the detector may be discriminated 

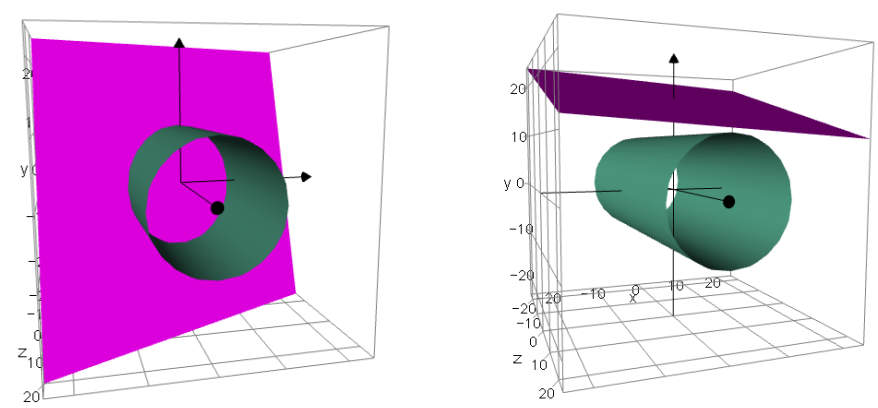

Figure 2. Visualization of two exemplary orientations of the recorded decay plane (violet) with respect to the cylindrical annihilation chamber (green). Left: in the case of a genuine o-Ps $\rightarrow 3 \gamma$ decay occurring in the positronium production medium located in the cylinder wall, the point of closest approach of the plane to detector center is small. Right: in case of a decay plane resulting from an accidental 3-photon coincidence, the decay plane may be distant from the center.

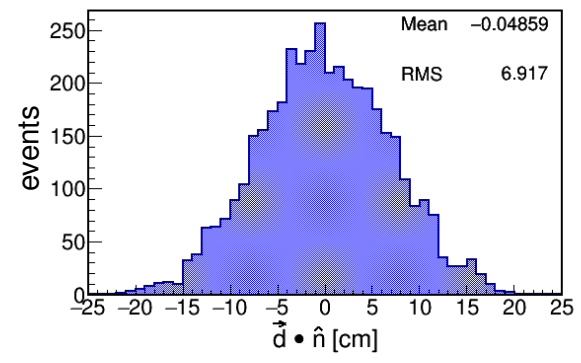

Figure 3. Distribution of the shortest distance $(d)$ between the o-Ps decay plane and the center of the J-PET detector (see Fig. 2), calculated as a projection to the normal of the decay plane $(\hat{n})$. Extreme values allow for rejection of artificial decay planes obtained for accidental $3 \gamma$ coincidences.

on the basis of the shortest distance of closest approach of the reconstructed $3 \gamma$ annihilation plane to the central point of the detector as demonstrated in Fig. 2. Distribution of such shortest distances for the selected $3 \gamma$ annihilation event candidates is presented in Fig. 3 . A part of artificial decay planes obtained from background events may be identified by the extreme values of this distance.

\section{Conclusions}

The possibility of using the J-PET detector to reconstruct the tomographic image of a cylindrical chamber using $2 \gamma$ annihilation events was demonstrated by an analysis performed using the procedures devised for tomographic imaging with J-PET. The selections of two-photon annihilation events were used in order to reconstruct lines of response (LORs) and difference between each photon time of flight which allowed to precisely find the annihilation point along a LOR. The trilateration based reconstruction method was successfully adapted to determine the location of the o-Ps annihilation point into three photons, which can be used to test CP and CPT invariance in such a purely leptonic system by searching for non-vanishing angular distribution correlations in the decays of o-Ps atoms.

This work was supported by The Polish National Center for Research and Development through grant INNOTECH-K1/IN1/64/159174/NCBR/12, the Foundation for Polish Science through the MPD and TEAM/2017-4/39 programmes, the National Science Centre of Poland through grants no. 2016/21/B/ST2/01222, 2017/25/N/NZ1/00861, the Ministry for Science and Higher Education through grants no. 6673/IA/SP/2016, 7150/E- 338/SPUB/2017/1, 7150/E-338/M/2017, 7150/E-338/M/2018 and the Austrian Science Fund FWF-P26783. 


\section{References}

[1] P. Moskal et al., Nucl. Instrum. Meth. A 764, 317 (2014)

[2] P. Moskal et al., Nucl. Instrum. Meth. A 775, 54 (2015)

[3] P. Moskal et al., Acta Phys. Polon. B 47, 509 (2016)

[4] S. Niedźwiecki et al., Acta Phys. Polon. B 48, 1567 (2017)

[5] P. Kowalski et al., Phys. Med. Biol. 63, 165008 (2018)

[6] L. Raczyński et al., Phys. Med. Biol. 62, 5076 (2017)

[7] P. Moskal et al., Phys. Med. Biol. 61, 2025 (2016)

[8] D. Kamińska et al., Eur. Phys. J. C 76 no.8, 445 (2016)

[9] M. Mohammed et al., Acta Phys. Polon. A 132, 1486 (2017)

[10] E. Czerwiński et al., Acta Phys. Polon. B 48, 1961 (2017)

[11] A. Gajos et al., Nucl. Instrum. Meth. A 819, 54 (2016)

[12] A. Gajos et al., Advances in High Energy Physics (in print), arXiv:1804.07148 [physics.ins-det] 